\title{
Impact of Venous Thromboembolism on the Formation and Progression of Carotid Atherosclerosis: The Tromsø Study
}

\author{
Caroline Lind $^{1,2}$ Birgit Småbrekke ${ }^{1} \quad$ Ludvig Balteskard Rinde ${ }^{1} \quad$ Kristian Hindberg ${ }^{1}$ \\ Ellisiv Bøgeberg Mathiesen ${ }^{1,3,4}$ Stein Harald Johnsen ${ }^{3,4}$ Kjell Arne Arntzen ${ }^{3,4}$ Inger Njølstad ${ }^{1,5}$ \\ Willem Lijfering ${ }^{6}$ Sigrid Kufaas Brækkan ${ }^{1,2}$ John-Bjarne Hansen ${ }^{1,2}$
}

${ }^{1}$ K.G. Jebsen Thrombosis Research and Expertise Center (TREC), Department of Clinical Medicine, UiT - The Arctic University of Norway, Tromsø, Norway

${ }^{2}$ Division of Internal Medicine, University Hospital of North Norway, Tromsø, Norway

${ }^{3}$ Brain and Circulation Research Group, Department of Clinical

Medicine, UiT - The Arctic University of Norway, Tromsø, Norway

${ }^{4}$ Department of Neurology and Clinical Neurophysiology, University

Hospital of North Norway, Tromsø, Norway

${ }^{5}$ Epidemiology of Chronic Diseases Research Group, Department of Community Medicine, UiT - The Arctic University of Norway,

Tromsø, Norway

${ }^{6}$ Department of Clinical Epidemiology, Leiden University Medical

Center, Leiden, The Netherlands

TH Open 2017;1:e66-e72.
Address for correspondence Caroline Lind, MD, K.G. Jebsen Thrombosis Research and Expertise Center (TREC), Department of Clinical Medicine, Faculty of Health Sciences, UiT - The Arctic University of Norway, N-9037 Tromsø, Norway (e-mail: caroline.lind@uit.no).

\begin{abstract}
Keywords

- atherosclerosis

- epidemiological studies

- risk factors

- venous thrombosis

Venous thromboembolism (VTE) is associated with increased risk of arterial cardiovascular diseases (CVD), and development of atherosclerosis secondary to VTE may be an intermediate between VTE and CVD. Therefore, we aimed to investigate whether incident VTE was associated with subsequent carotid atherosclerosis formation and progression in a population-based observational study. Subjects attending two or more ultrasound examinations of the right carotid artery, with measurement of total plaque area (TPA), in the Tromsø Study in 1994-1995, 2001-2002, and/or 2007-2008 were eligible. We identified 150 subjects diagnosed with first-lifetime VTE between the initial and follow-up visit, and randomly selected 600 age- and sex-matched subjects without VTE between the visits. Subjects with VTE and carotid plaque(s) at the first visit had 4.1 $\mathrm{mm}^{2}(\beta: 4.13,95 \% \mathrm{Cl}:-1.72$ to 9.98$)$ larger change in TPA between the first and second visit compared with subjects without VTE after adjustment for change in highsensitivity C-reactive protein (hs-CRP) and traditional atherosclerotic risk factors. The association remained after restricting the analyses to VTE events diagnosed in the first half of the time interval between the carotid ultrasounds $(\beta: 4.02,95 \% \mathrm{Cl}$ : -3.66 to 11.70), supporting that the change in TPA occurred subsequent to the VTE. No association was found between VTE and novel carotid plaque formation. In conclusion, we found a possible association between VTE and atherosclerosis progression in those with already established carotid plaques, but not between VTE and novel plaque formation. The association between VTE and carotid plaque progression was not mediated by low-grade inflammation assessed by hs-CRP.
\end{abstract}

DOI https://doi.org/ 10.1055/s-0037-1603983. ISSN 2512-9465.
(C) 2017 Georg Thieme Verlag KG Stuttgart $\cdot$ New York
License terms

(c) (1) $\ominus$ (\$) 


\section{Introduction}

Population-based cohorts and registry-based studies have shown that venous thromboembolism (VTE) is associated with future risk of arterial cardiovascular diseases (CVD) such as myocardial infarction (MI) and ischemic stroke. ${ }^{1,2}$ The possible mechanisms behind this relationship remain unsettled, but atherosclerosis may play a key role, as atherosclerosis is a major risk factor for arterial CVD. ${ }^{3,4}$ A higher prevalence of atherosclerosis has been reported in patients with unprovoked VTE compared with control subjects. ${ }^{5,6}$ However, these studies were not designed to explore the temporal sequence between VTE and subsequent atherosclerosis.

To the best of our knowledge, no study has examined the association between incident VTE and risk of future formation and progression of carotid atherosclerosis in a general population. A VTE event is often followed by an abrupt and prolonged increase in levels of inflammatory markers, including C-reactive protein (CRP), ${ }^{7,8}$ and sustained elevation of CRP has been related to the development and progression of atherosclerosis. ${ }^{9}$ We hypothesized that inflammatory processes secondary to VTE could promote formation and progression of carotid atherosclerosis. Therefore, we set out to investigate whether an incident VTE was associated with subsequent formation and progression of carotid atherosclerosis, assessed by total plaque area (TPA), and whether this possible association was mediated by low-grade inflammation, assessed by high-sensitivity CRP (hs-CRP) in a population-based matched cohort study.

\section{Materials and Methods}

\section{Study Population}

Participants were recruited from the fourth, fifth, and sixth surveys of the Tromsø Study, conducted in 1994-1995, 2001-2002, and 2007-2008, respectively. The Tromsø Study is a large population-based cohort study. ${ }^{10}$ All inhabitants aged $\geq 25$ years in the municipality of Tromsø, Norway, were invited to the fourth survey (Tromsø 4). All subjects aged 55 to 74 years, and 5 to $10 \%$ of subjects in the remaining 5 -year age groups, were invited to a more extensive screening visit, including ultrasound examination of the right carotid artery. Subjects attending the extensive screening in Tromsø 4 who were still alive and had not moved from Tromsø were reinvited to participate in the fifth and sixth surveys of the Tromsø Study (Tromsø 5 and 6, respectively).

In addition, random samples within different age groups of the Tromsø population were invited to Tromsø 5 and 6 . In total, 6,688, 5,422, and 7,083 subjects were scanned with ultrasound of the right carotid artery in Tromsø 4, 5, and 6, respectively. Of these, 5,479 subjects attended two or more surveys and were considered eligible for our study. Subjects with VTE before their first survey $(n=19)$ and subjects with missing values of TPA $(n=92)$ were excluded. We identified 150 subjects who were diagnosed with incident VTE between ultrasound examinations. The identification and validation of VTE events in the Tromsø Study has been described in detail elsewhere and included a combination of symptoms and objective diagnostics. ${ }^{11}$ The survey inter- vals were between the first and second visits (i.e., the ultrasounds in Tromsø 4 and 5, 5 and 6, and/or 4 and 6), and VTE patients attending all three surveys contributed with two-survey intervals. For each VTE patient, we randomly selected four subjects matched for sex and age in the 5-year age groups who attended the same survey intervals, but were not diagnosed with incident VTE between the surveys. In total, 750 subjects were included in our matched cohort. The study was approved by the Regional Committee of Medical and Health Research Ethics, North Norway, and all subjects gave their informed written consent to participate.

\section{Baseline Measurements}

Baseline information was collected by self-administered questionnaires, blood samples, and physical examinations. Questionnaires were used to obtain information on current smoking, diabetes mellitus, history of MI and stroke, and use of lipid-lowering drugs. Height, weight, blood pressure, and non-fasting serum lipids were measured as previously described. ${ }^{12}$ hs-CRP was measured after storage at $-70^{\circ} \mathrm{C}$ in Troms $\varnothing 4$ and $-20^{\circ} \mathrm{C}$ in Troms $\varnothing 5$ and 6 , by a particle-enhanced immunoturbidimetric assay on a Modular P(Tromsø 4 and 6) or Hitachi 917 (Tromsø 5) autoanalyzer (Roche Hitachi, Mannheim, Germany), using reagents from Roche Diagnostics (Mannheim). ${ }^{13}$ Samples from Tromsø 4 were analyzed after 12 years of storage, and samples from Tromsø 5 and 6 were analyzed in batches at the time of the surveys. In Tromsø 6, hsCRP was measured at two different time points, and if both measurements were available, the average was recorded. The lower detection limit of the hs-CRP assay was $0.03 \mathrm{mg} / \mathrm{L}$. Body mass index (BMI) was calculated as weight in kilograms divided by the square of height in meters $\left(\mathrm{kg} / \mathrm{m}^{2}\right)$.

\section{Ultrasound Examinations}

High-resolution B-mode and color/pulsed-wave Doppler ultrasonography of the right carotid artery were performed by trained personnel with the use of an ultrasound scanner (Acuson XP10 128 ART equipped with a 7.5-MHz linear transducer in Tromsø 4 and 5 and GE Vivid 7 with a linear 12-MHz transducer in Tromsø 6), as previously described in detail. ${ }^{14-16}$ Plaques were registered in the near and far walls of the common carotid artery (CCA), the bifurcation, and the internal carotid artery (ICA; six locations). A plaque was defined as a local protrusion of the vessel wall into the lumen of $50 \%$ or more compared with adjacent intima media thickness (IMT). For each plaque, a still image was recorded and digitized using the Matrix Meteor II frame-grabber and Matrox Intellicam. Adobe Photoshop 7.0 was subsequently used to measure plaque areas by outlining the perimeter of each plaque with a cursor, and the plaque area was calculated as pixel values. For the resolution used in this study, a plaque area of 167 pixels corresponded to $1 \mathrm{~mm}^{2}$. In subjects with more than one plaque, the TPA was calculated as the sum of all plaque areas. Novel plaque formation was defined as the development of new plaques at the second visit in vessels without plaques at the first visit. Plaque progression was defined as an increase in TPA between the first and second visits. Previous publications from the Tromsø Study have 
demonstrated substantial between- and within-sonographer reproducibility ${ }^{15}$ and excellent computer-assisted offline classification for carotid plaques. ${ }^{17}$

TPA was chosen as the preferred measure of atherosclerosis because a meta-analysis of 11 population-based studies showed that measurement of carotid plaque characteristics (presence/absence, plaque thickness, and plaque area) had a higher accuracy for predicting arterial CVD than IMT. ${ }^{18}$ Moreover, prospective cohorts reported that carotid plaques, but not carotid IMT, were predictors of coronary heart disease. ${ }^{19,20}$

\section{Statistical Analysis}

Statistical analyses were performed using STATA version 14.0 (Stata Corporation, College Station, Texas, United States). Logistic regression models were used to calculate odds ratios (ORs) with 95\% confidence intervals (CIs) for novel plaque formation and plaque progression (yes/no) according to VTE exposure. In subjects with carotid plaque(s) at first visit, multiple linear regression models were applied to compare the change in TPA between the first and second visits according to VTE status. Annual change in TPA between the first and second visits was used as the outcome in a subanalysis. Moreover, TPA at the second visit was square root transformed to approximate normal distribution and compared in subjects with and without VTE using multiple linear regression. Adjustments in Model 1 included age, sex, and study interval by matching. Model 2 included, in addition to Model 1, change in hs-CRP between the first and second visits. In Model 3, variables previously shown to be associated with carotid atherosclerosis in the Tromsø Study (i.e., systolic blood pressure, total cholesterol, high-density lipoprotein cholesterol, BMI, self-reported current smoking, and diabetes mellitus) ${ }^{14,21}$ were additionally added.

Because the VTE events occurred between two visits, the temporal sequence of the atherosclerosis progression and the VTE could not be completely determined. However, if the VTE occurred early in the time interval, the change in TPA would more likely occur subsequent to the VTE. To model this time sequence, we performed a subanalysis restricted to VTE events diagnosed in the first period of the interval between the carotid ultrasound measurements.

For sensitivity purposes, all analyses were re-run after exclusion of subjects with self-reported history of MI or stroke at the first visit. Statistical interactions between VTE and age, VTE and sex, and VTE and BMI were tested by including cross-product terms in the regression models. The cross-product terms were centered at the mean of the covariate to reduce multicollinearity. Correlations were assessed by Spearman's rank correlation, and model assumptions were evaluated by residual analyses.

\section{Results}

- Table 1 displays the characteristics of the study participants at the first visit and change in BMI, total cholesterol, and TPA between the first and second visits. Subjects diagnosed with VTE had slightly higher mean BMI, higher change in BMI and hs-CRP, comprised a higher proportion of current smokers and subjects commencing lipid-lowering drugs, and a lower proportion of subjects with previous CVD, plaques with low echogenicity, and plaques in the bifurcation and the ICA compared with those without VTE. Moreover, subjects with VTE had higher TPA at the first and second visits, and a larger change in TPA between the visits compared with subjects without VTE. Among the VTE events, 64\% were deep vein thrombosis (DVTs) and 36\% were pulmonary embolisms (PEs), and $45 \%$ were classified as unprovoked (data not shown).

ORs of novel carotid plaque formation and carotid plaque progression according to incident VTE are shown in - Table 2. In Model 1, VTE was associated with 25\% increased risk of novel carotid plaque formation (OR: $1.25,95 \% \mathrm{CI}$ : 0.83-1.98) and $23 \%$ increased risk of carotid plaque progression (OR: 1.23, 95\% CI: 0.85-1.77). Further adjustments for change in hs-CRP attenuated the association between VTE and plaque formation (OR: 1.04, 95\% CI: 0.67-1.61), but had a negligible effect on the association between incident VTE and carotid plaque progression (OR: 1.12, 95\% CI: 0.77-1.62). Similar results were obtained after excluding subjects with previous CVD in sensitivity analyses and after further adjustment for IMT (novel plaque) or TPA (plaque progression) at the first visit (data not shown).

To assess the impact of VTE on carotid plaque progression in subjects with plaque(s) at baseline, multiple linear regression models were applied to those with one or more carotid plaque(s) at the first visit $(n=373)$. - Table 3 shows the mean values and regression coefficients $(\beta s)$ for change in TPA between the first and second visits and TPA at the second visit according to VTE. Among subjects with established carotid plaque(s), there was no association between VTE and TPA at the second visit in any of the multiple linear regression models. Those with VTE had $2.7-\mathrm{mm}^{2}$ larger change in TPA between the first and second visits compared with those without VTE in Model 1 ( $\beta$ : $2.70,95 \% \mathrm{CI}$ : -2.96 to 8.35). Further adjustments for change in hs-CRP between the visits did not alter the result, whereas VTE was associated with a $4.1-\mathrm{mm}^{2}$ larger change in TPA after additionally taking other traditional atherosclerotic risk factors into consideration (Model 3: $\beta=4.13,95 \% \mathrm{CI}=-1.72$ to 9.98). Comparable results were found when the annual change in TPA from the first to the second visit was used as the outcome and when analyses were further adjusted for TPA and plaque echogenicity at the first visit and initiation of lipid-lowering therapy between the first and second visits. Moreover, exclusion of subjects with previous MI or stroke did not alter the results (data not shown).

When analyses were restricted to VTE events diagnosed in the first half of the time interval between the carotid ultrasounds, no association was found between VTE and novel carotid plaque formation (multivariable OR: $0.57,95 \% \mathrm{CI}$ : $0.27-1.20$ ) or carotid plaque progression (OR: $0.99,95 \% \mathrm{CI}$ : $0.58-1.67)$. Moreover, in subjects with carotid plaque(s) at the first visit, VTE was associated with an increase in TPA ( $\beta$ : $4.02,95 \% \mathrm{CI}:-3.66$ to 11.70 ), but did not predict mean TPA at the second visit ( $\beta$ : $0.61,95 \% \mathrm{CI}:-0.33$ to 1.56$)$ after adjustment for change in hs-CRP and traditional atherosclerotic risk factors. 
Table 1 Characteristics of participants

\begin{tabular}{|c|c|c|}
\hline & VTE $(n=150)$ & No VTE $(n=600)$ \\
\hline Age & $62.4 \pm 6.8$ & $62.4 \pm 6.9$ \\
\hline Sex (men) & $77(51.3)$ & $308(51.3)$ \\
\hline Follow-up time $(y)$ & $9.4 \pm 3.3$ & $9.4 \pm 3.3$ \\
\hline Systolic blood pressure (mm Hg) & $144.1 \pm 20.4$ & $143.9 \pm 20.5$ \\
\hline Total cholesterol (mmol/L) & $6.65 \pm 1.27$ & $6.70 \pm 1.15$ \\
\hline Change in total cholesterol $(\mathrm{mmol} / \mathrm{L})^{\mathrm{a}}$ & $-0.81 \pm 1.36$ & $-0.74 \pm 1.22$ \\
\hline High-density lipoprotein cholesterol (mmol/L) & $1.54 \pm 0.43$ & $1.55 \pm 0.43$ \\
\hline Body mass index $\left(\mathrm{kg} / \mathrm{m}^{2}\right)$ & $27.5 \pm 3.8$ & $26.1 \pm 3.6$ \\
\hline Change in body mass index $\left(\mathrm{kg} / \mathrm{m}^{2}\right)^{\mathrm{a}}$ & $0.8 \pm 2.4$ & $0.6 \pm 2.0$ \\
\hline Diabetes mellitus $^{\mathrm{b}}$ & $5(3.3)$ & $18(3.0)$ \\
\hline Current smoking ${ }^{\mathrm{b}}$ & $32(21.3)$ & $122(20.3)$ \\
\hline History of cardiovascular disease ${ }^{\mathrm{b}}$ & $7(4.7)$ & $39(6.5)$ \\
\hline Commenced lipid-lowering drugs ${ }^{\mathrm{a}, \mathrm{b}}$ & $36(27.1)$ & $118(21.3)$ \\
\hline High-sensitivity C-reactive protein (mg/L) & $2.66 \pm 3.57$ & $2.37 \pm 4.76$ \\
\hline Number with plaques & $70(46.7)$ & $303(50.5)$ \\
\hline 1 plaque & $37(24.7)$ & $194(32.3)$ \\
\hline 2 plaques & $23(15.3)$ & $78(13.0)$ \\
\hline$\geq 3$ plaques & $10(6.7)$ & $31(5.2)$ \\
\hline \multicolumn{3}{|l|}{ Plaque location(s) } \\
\hline Common carotid artery & $8(5.3)$ & $20(3.3)$ \\
\hline Carotid bifurcation & $63(42.0)$ & $260(43.3)$ \\
\hline Internal carotid artery & $21(14.0)$ & $95(15.8)$ \\
\hline Mean intima media thickness (mm) & $0.85 \pm 0.15$ & $0.85 \pm 0.15$ \\
\hline Total plaque area $\left(\mathrm{mm}^{2}\right)$ & $10.03 \pm 17.86$ & $9.12 \pm 13.17$ \\
\hline Total plaque area $\left(\mathrm{mm}^{2}\right)$ 2nd visit & $21.29 \pm 28.45$ & $18.08 \pm 22.08$ \\
\hline Change in total plaque area $\left(\mathrm{mm}^{2}\right)^{a}$ & $11.26 \pm 18.99$ & $8.96 \pm 18.51$ \\
\hline Low plaque echogenicity & $57(38.0)$ & $246(41.0)$ \\
\hline
\end{tabular}

Notes: Characteristics of participants at the first visit according to venous thromboembolism (VTE) status between the first and second visits: the Tromsø Study. Values are numbers (\%) or mean \pm standard deviation.

a Between first and second visits.

bSelf-reported.

Table 2 Impact of VTE on novel carotid plaque formation and plaque progression

\begin{tabular}{|c|c|c|c|c|c|}
\hline & & & $\begin{array}{l}\text { Model } 1 \\
\text { OR }(95 \% \mathrm{Cl})\end{array}$ & $\begin{array}{l}\text { Model } 2 \\
\text { OR }(95 \% \mathrm{Cl})\end{array}$ & $\begin{array}{l}\text { Model } 3 \\
\text { OR }(95 \% \mathrm{Cl})\end{array}$ \\
\hline New plaque & Yes & No & & & \\
\hline VTE - & 135 & 465 & Ref. & Ref. & Ref. \\
\hline VTE + & 40 & 110 & $1.25(0.83-1.89)$ & $1.04(0.67-1.61)$ & $1.12(0.72-1.75)$ \\
\hline Plaque progression & Yes & No & & & \\
\hline VTE - & 334 & 266 & Ref. & Ref. & Ref. \\
\hline VTE + & 91 & 59 & $1.23(0.85-1.77)$ & $1.12(0.77-1.62)$ & $1.19(0.81-1.75)$ \\
\hline
\end{tabular}

Note: Odds ratios (ORs) with $95 \%$ confidence intervals (Cls) of novel carotid plaque formation and plaque progression according to incident venous thromboembolism (VTE): the Tromsø Study.

Model 1: adjusted for age, sex, and study interval by matching.

Model 2: additionally adjusted for change in high-sensitivity C-reactive protein from first to second visits.

Model 3: additionally adjusted for systolic blood pressure, total cholesterol, high-density lipoprotein cholesterol, diabetes mellitus, body mass index, and current smoking. 
Table 3 Impact of VTE on total plaque area

\begin{tabular}{|c|c|c|c|c|}
\hline & Mean \pm SD & $\beta$ model 1 & $\beta$ model 2 & $\beta$ model 3 \\
\hline \multicolumn{5}{|l|}{$\triangle \mathrm{TPA}\left(\mathrm{mm}^{2}\right)$} \\
\hline VTE $-(n=303)$ & $9.11 \pm 21.62$ & Ref. & Ref. & Ref. \\
\hline $\mathrm{VTE}+(n=70)$ & $11.81 \pm 22.29$ & $2.70(-2.96$ to 8.35$)$ & $2.70(-3.04$ to 8.43$)$ & $4.13(-1.72$ to 9.98$)$ \\
\hline \multicolumn{5}{|l|}{ TPA $\left(\mathrm{mm}^{2}\right)$} \\
\hline $\mathrm{VTE}-(n=303)$ & $27.17 \pm 24.23$ & Ref. & Ref. & Ref. \\
\hline $\mathrm{VTE}+(n=70)$ & $33.30 \pm 34.52$ & $0.22(-0.49 \text { to } 0.93)^{\mathrm{a}, \mathrm{b}}$ & $0.27(-0.45 \text { to } 0.99)^{\mathrm{a}, \mathrm{b}}$ & $0.43(-0.29 \text { to } 1.16)^{a, b}$ \\
\hline
\end{tabular}

Notes: Mean values with standard deviations (SDs) and regression coefficients ( $\beta \mathrm{s}$ ) with $95 \%$ confidence intervals ( $\mathrm{Cls}$ ) for change in total plaque area $(\triangle T P A)$ and TPA at the second visit according to incident venous thromboembolism (VTE) in subjects with carotid plaque(s) at the first visit: the Tromsø Study.

Model 1: adjusted for age, sex, and study interval by matching.

Model 2: additionally adjusted for change in high-sensitivity C-reactive protein from first to second visits.

Model 3: additionally adjusted for systolic blood pressure, total cholesterol, high-density lipoprotein cholesterol, diabetes mellitus, body mass index, and current smoking.

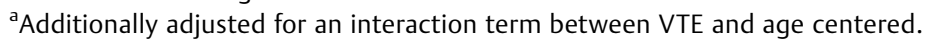

bSquare root transformed TPA at second visit.

\section{Discussion}

In this matched cohort study, we investigated whether incident VTE was associated with the formation and progression of carotid atherosclerosis and to what extent a possible association was mediated by low-grade inflammation. Subjects with carotid plaque(s) at the first visit and VTE had a 4.1- $\mathrm{mm}^{2}$ larger increase in TPA compared with those without VTE after adjustment for traditional atherosclerotic risk factors, including CRP. The apparent association between incident VTE and plaque progression in subjects with carotid plaque(s) remained unchanged after restricting the analyses to VTE events diagnosed in the first half of the time interval between the carotid ultrasounds. In contrast, VTE was not associated with the formation of new atherosclerotic plaques. Our findings may suggest that VTE accelerates plaque progression independently of traditional atherosclerotic risk factors and low-grade inflammation in those who have already developed plaques. These findings must, however, be interpreted with caution due to limited statistical power, and replicated in larger cohorts.

Recently, we reported that incident VTE was associated with subsequent risk of MI and ischemic stroke in a large population-based cohort. ${ }^{1}$ An interrelation between VTE and carotid atherosclerosis has previously been demonstrated in case-control studies, in which subjects with unprovoked DVT ${ }^{5}$ and $\mathrm{VTE}^{6,22}$ had an increased prevalence of asymptomatic carotid atherosclerosis. The latter studies were not designed to explore the temporal sequence between VTE and atherosclerosis. To the best of our knowledge, this study is the first designed to model the time-sequence between incident VTE and carotid atherosclerosis. We found that incident VTE was associated with an increase in TPA in subjects who already had carotid plaque(s). The possible increase in TPA by VTE in subjects with carotid plaque(s) was strengthened in multivariable analyses adjusted for traditional atherosclerotic risk factors, specifically BMI and smoking, and was consistent when restricted to VTE events diagnosed in the first half of the time-interval between the carotid ultrasounds. Furthermore, the possible association between VTE and carotid plaque progression in those with established atherosclerosis remained after taking plaque size, plaque echogenicity, and use of lipid-lowering drugs into consideration. We have previously shown that the risk of MI increased by $23 \%$ per $2.4 \mathrm{~mm}^{2}$ increase in TPA after adjusting for traditional atherosclerotic risk factors. ${ }^{23}$ Extrapolated to the present study population, this may suggest that subjects with established carotid plaque(s) and VTE have $42 \%$ increased risk of MI in our population. However, our study was limited by a low statistical power. Post hoc analysis revealed that we only had a statistical power of 0.29 for the association between incident VTE and change in TPA in the multivariable model, which implies a high chance (71\%) of rejecting our hypothesis of a true association. To achieve a sufficient power of 0.80 given the effect size and variance we experienced, the study should have included nearly four times as many participants with carotid plaque(s) at the first visit $(n=1,480)$. Thus, our findings should be interpreted with caution, and be considered as hypothesis generating, and encourage replication studies to be performed to further test the hypothesis.

Previous studies have shown that VTE is accompanied by both an acute ${ }^{7,24}$ and a long-term ${ }^{8}$ inflammatory state with elevated levels of inflammatory markers including CRP. In a cohort study, hs-CRP levels were significantly twofold higher 5 years after an unprovoked DVT compared with subjects without VTE. ${ }^{8}$ Furthermore, inflammation, assessed by high CRP levels, has been recognized as a risk factor for arterial thrombotic diseases ${ }^{25-27}$ with a nearly linear relationship with arterial CVD risk. ${ }^{27}$ Therefore, chronic low-grade inflammation following a VTE may mediate an association between venous and arterial thrombotic diseases.

Conflicting results have, however, been reported regarding the association between CRP and future carotid atherosclerosis. In our study, hs-CRP did not contribute to the apparent association between incident VTE and plaque progression in 
subjects with carotid plaque(s). Some observational studies have reported that hs-CRP was not associated with carotid IMT progression $^{28}$ or plaque presence, ${ }^{29}$ and recent findings from the Tromsø Study showed that hs-CRP did not predict carotid plaque progression (Agnethe Eltoft, MD, unpublished data, 2016). In contrast, other prospective cohorts have shown that high CRP independently predicted atherosclerosis progression, ${ }^{30-32}$ and CRP in the highest ( $>2.9 \mathrm{mg} / \mathrm{L}$ ) compared with the lowest $(\leq 0.8 \mathrm{mg} / \mathrm{L}$ ) quartile was associated with 4.5 -fold increased risk of atherosclerosis progression in a cohort of subjects 55 years or older. $^{31}$ Moreover, the severity and progression of extracranial atherosclerosis increased with increasing CRP levels after adjusting for atherosclerotic risk factors, ${ }^{32}$ and both baseline and change in hs-CRP were significantly associated with progression of atherosclerosis ${ }^{30}$ in cohort studies. Consequently, it is unknown whether the apparent lack of effect of hs-CRP on the possible association between VTE and progression of carotid atherosclerosis is due to an actual lack of effect of CRP on carotid plaque progression, or whether the effect of VTE on chronic inflammation, assessed by hs-CRP, is too small to promote plaque progression. Our findings suggest, however, that a possible association between incident VTE and carotid plaque progression is independent of the inflammatory response assessed by hs-CRP.

Potentially, the association between VTE and carotid plaque progression could be explained by shared risk factors, such as thrombophilia, hyperhomocysteinemia, or microalbuminuria. ${ }^{33-35}$ However, factor V Leiden and hyperhomocysteinemia did not explain the association between unprovoked DVT and carotid atherosclerosis in a case-control study. ${ }^{5}$ A hypercoagulable state or endothelial dysfunction following the VTE event may contribute to the observed increase in TPA in subjects with VTE. Concordantly, the procoagulant factor fibrinogen is associated with progression of carotid atherosclerosis, ${ }^{36}$ and levels of coagulation factors VII and XII have been shown to influence the size and stability of carotid plaques in mice. ${ }^{37}$ Furthermore, levels of circulating markers of endothelial dysfunction, such as the von Willebrand factor antigen, were significantly higher after 5 years in acute DVT patients compared with controls without VTE in a prospective cohort. ${ }^{8}$

The main strengths of our study include that the study participants were recruited from a general population with wide age distribution, high participation rate, and thoroughly validated events of VTE. The study has some limitations. The time intervals between the carotid ultrasound surveys were wide, and it is unknown at what time the change in subclinical atherosclerosis measures arose. Therefore, the change in the atherosclerosis measures could potentially have occurred before the VTE events. However, a similar increase in TPA was found in subjects with carotid plaque(s) after restricting the analyses to VTE events diagnosed in the first half of the time interval between the carotid ultrasounds, supporting a temporal sequence between VTE and subsequent carotid plaque progression. Furthermore, hs-CRP was measured in serum after storage at $-70^{\circ} \mathrm{C}$ for 12 years, which may have affected the hs-CRP levels. However, storage of frozen plasma samples over time has previously been shown to have a negligible influence on the assayed values of biomarkers including CRP. ${ }^{38}$ Only subjects able to attend at least two carotid ultrasounds were included in our study. Although the attendance rate was high in our study, nonresponse bias is a possible limitation, because those who participate in cohort studies tend to be healthier than the general population. The statistical power in our study was low, and thereby we potentially failed to achieve significance of a true association between incident VTE and carotid atherosclerosis because too few study participants were included. Therefore, replication studies are warranted to verify or reject our findings.

In conclusion, our study suggests that VTE is associated with a possible increase in carotid plaque progression in those who already have atherosclerosis, but not with novel plaque formation. The association between VTE and carotid plaque progression was not mediated by low-grade inflammation, assessed by hs-CRP.

Conflict of Interest

None declared.

Funding

This work was supported by Stiftelsen Kristian Gerhard Jebsen and the Northern Norway Regional Health Authority.

\section{References}

1 Lind C, Flinterman LE, Enga KF, et al. Impact of incident venous thromboembolism on risk of arterial thrombotic diseases. Circulation 2014;129(08):855-863

2 Sørensen HT, Horvath-Puho E, Pedersen L, Baron JA, Prandoni P. Venous thromboembolism and subsequent hospitalisation due to acute arterial cardiovascular events: a 20-year cohort study. Lancet 2007;370(9601):1773-1779

3 van den Oord SC, Sijbrands EJ, ten Kate GL, et al. Carotid intimamedia thickness for cardiovascular risk assessment: systematic review and meta-analysis. Atherosclerosis 2013;228(01):1-11

4 Davidsson L, Fagerberg B, Bergström G, Schmidt C. Ultrasoundassessed plaque occurrence in the carotid and femoral arteries are independent predictors of cardiovascular events in middle-aged men during 10 years of follow-up. Atherosclerosis 2010;209(02): 469-473

5 Prandoni P, Bilora F, Marchiori A, et al. An association between atherosclerosis and venous thrombosis. N Engl J Med 2003; 348(15):1435-1441

6 Jezovnik MK, Poredos P, Lusa L. Idiopathic venous thrombosis is associated with preclinical atherosclerosis. J Atheroscler Thromb 2010;17(03):304-311

7 Roumen-Klappe EM, den Heijer M, van Uum SH, van der VenJongekrijg J, van der Graaf F, Wollersheim $\mathrm{H}$. Inflammatory response in the acute phase of deep vein thrombosis. J Vasc Surg 2002;35(04):701-706

8 Jezovnik MK, Fareed J, Poredos P. Subjects with a history of idiopathic deep venous thrombosis have long-term increased levels of inflammatory markers and markers of endothelial damage. Clin Appl Thromb Hemost 2017;23(02):124-131

9 Zhang YX, Cliff WJ, Schoefl GI, Higgins G. Coronary C-reactive protein distribution: its relation to development of atherosclerosis. Atherosclerosis 1999;145(02):375-379 
10 Jacobsen BK, Eggen AE, Mathiesen EB, Wilsgaard T, Njølstad I. Cohort profile: the Tromso Study. Int J Epidemiol 2012;41(04):961-967

11 Braekkan SK, Borch KH, Mathiesen EB, Njølstad I, Wilsgaard T, Hansen JB. Body height and risk of venous thromboembolism: the Tromsø Study. Am J Epidemiol 2010;171(10):1109-1115

12 Brækkan SK, Hald EM, Mathiesen EB, et al. Competing risk of atherosclerotic risk factors for arterial and venous thrombosis in a general population: the Tromso study. Arterioscler Thromb Vasc Biol 2012;32(02):487-491

13 Horvei LD, Grimnes G, Hindberg K, et al. C-reactive protein, obesity, and the risk of arterial and venous thrombosis. J Thromb Haemost 2016;14(08):1561-1571

14 Johnsen SH, Mathiesen EB, Fosse E, et al. Elevated high-density lipoprotein cholesterol levels are protective against plaque progression: a follow-up study of 1952 persons with carotid atherosclerosis the Tromsø study. Circulation 2005;112(04):498-504

15 Joakimsen O, Bønaa KH, Stensland-Bugge E. Reproducibility of ultrasound assessment of carotid plaque occurrence, thickness, and morphology. The Tromsø Study. Stroke 1997;28(11):2201-2207

16 Stensland-Bugge E, Bønaa KH, Joakimsen O. Reproducibility of ultrasonographically determined intima-media thickness is dependent on arterial wall thickness. The Tromsø Study. Stroke 1997;28(10):1972-1980

17 Fosse E, Johnsen SH, Stensland-Bugge E, et al. Repeated visual and computer-assisted carotid plaque characterization in a longitudinal population-based ultrasound study: the Tromsø study. Ultrasound Med Biol 2006;32(01):3-11

18 Inaba Y, Chen JA, Bergmann SR. Carotid plaque, compared with carotid intima-media thickness, more accurately predicts coronary artery disease events: a meta-analysis. Atherosclerosis 2012; 220(01):128-133

19 Plichart M, Celermajer DS, Zureik M, et al. Carotid intima-media thickness in plaque-free site, carotid plaques and coronary heart disease risk prediction in older adults. The Three-City Study. Atherosclerosis 2011;219(02):917-924

20 Johnsen SH, Mathiesen EB, Joakimsen O, et al. Carotid atherosclerosis is a stronger predictor of myocardial infarction in women than in men: a 6 -year follow-up study of 6226 persons: the Tromsø Study. Stroke 2007;38(11):2873-2880

21 Herder M, Johnsen SH, Arntzen KA, Mathiesen EB. Risk factors for progression of carotid intima-media thickness and total plaque area: a 13-year follow-up study: the Tromsø Study. Stroke 2012; 43(07):1818-1823

22 Milan M, Vedovetto V, Bilora F, Pesavento R, Prandoni P. Further evidence in support of the association between venous thrombosis and atherosclerosis: a case-control study. Thromb Res 2014; 134(05):1028-1031

23 Hald EM, Lijfering WM, Mathiesen EB, et al. Carotid atherosclerosis predicts future myocardial infarction but not venous thromboembolism: the Tromso study. Arterioscler Thromb Vasc Biol 2014;34(01):226-230

24 Rabinovich A, Cohen JM, Cushman M, Kahn SR; BioSOX Investigators. Association between inflammation biomarkers, anatomic extent of deep venous thrombosis, and venous symptoms after deep venous thrombosis. J Vasc Surg Venous Lymphat Disord 2015;3(04):347-53.e1

25 Buckley DI, Fu R, Freeman M, Rogers K, Helfand M. C-reactive protein as a risk factor for coronary heart disease: a systematic review and meta-analyses for the U.S. Preventive Services Task Force. Ann Intern Med 2009;151(07):483-495

26 Ridker PM, Cushman M, Stampfer MJ, Tracy RP, Hennekens CH. Inflammation, aspirin, and the risk of cardiovascular disease in apparently healthy men. N Engl J Med 1997;336(14):973-979

27 Kaptoge S, Di Angelantonio E, Lowe G, et al; Emerging Risk Factors Collaboration. C-reactive protein concentration and risk of coronary heart disease, stroke, and mortality: an individual participant meta-analysis. Lancet 2010;375(9709):132-140

28 Willeit P, Thompson SG, Agewall S, et al; PROG-IMT Study Group. Inflammatory markers and extent and progression of early atherosclerosis: Meta-analysis of individual-participant-data from 20 prospective studies of the PROG-IMT collaboration. Eur J Prev Cardiol 2016;23(02):194-205

29 Halvorsen DS, Johnsen SH, Mathiesen EB, Njølstad I. The association between inflammatory markers and carotid atherosclerosis is sex dependent: the Tromsø Study. Cerebrovasc Dis 2009; 27(04):392-397

30 Schillinger M, Exner M, Mlekusch W, et al. Inflammation and Carotid Artery-Risk for Atherosclerosis Study (ICARAS). Circulation 2005;111(17):2203-2209

31 van der Meer IM, Iglesias del Sol A, Hak AE, Bots ML, Hofman A Witteman JC. Risk factors for progression of atherosclerosis measured at multiple sites in the arterial tree: the Rotterdam Study. Stroke 2003;34(10):2374-2379

32 Schmidt R, Schmidt H, Pichler M, et al. C-reactive protein, carotid atherosclerosis, and cerebral small-vessel disease: results of the Austrian Stroke Prevention Study. Stroke 2006;37(12):2910-2916

33 Ye Z, Liu EH, Higgins JP, et al. Seven haemostatic gene polymorphisms in coronary disease: meta-analysis of 66,155 cases and 91,307 controls. Lancet 2006;367(9511):651-658

34 den Heijer M, Rosendaal FR, Blom HJ, Gerrits WB, Bos GM. Hyperhomocysteinemia and venous thrombosis: a meta-analysis. Thromb Haemost 1998;80(06):874-877

35 Mahmoodi BK, Gansevoort RT, Veeger NJ, et al; Prevention of Renal and Vascular End-stage Disease (PREVEND) Study Group. Microalbuminuria and risk of venous thromboembolism. JAMA 2009;301(17):1790-1797

36 Chambless LE, Folsom AR, Davis V, et al. Risk factors for progression of common carotid atherosclerosis: the atherosclerosis risk in communities study, 1987-1998. Am J Epidemiol 2002;155(01):38-47

37 Kuijpers MJ, van der Meijden PE, Feijge MA, et al. Factor XII regulates the pathological process of thrombus formation on ruptured plaques. Arterioscler Thromb Vasc Biol 2014;34(08): 1674-1680

38 Lewis MR, Callas PW, Jenny NS, Tracy RP. Longitudinal stability of coagulation, fibrinolysis, and inflammation factors in stored plasma samples. Thromb Haemost 2001;86(06):1495-1500 